JOURNAL OF MODERN OPTICS, 2000, vOL. 47, NO. 11, 1877-1886

\title{
Propagation properties of non-paraxial spatial solitons
}

\author{
P. CHAMORRO-POSADA
}

Departamento de Teoría de la Señal y Comunicaciones e Ingeniería Telemática, ETSI Telecomunicación, Universidad de Valladolid, Campus Miguel Delibes, 47011 Valladolid, Spain

\author{
G. S. McDONALD广 and G. H. C. NEW \\ Laser Optics \& Spectroscopy Group, The Blackett Laboratory, \\ Imperial College of Science Technology and Medicine, \\ Prince Consort Road, London SW7 2BZ, UK
}

\section{(Received 6 October 1999)}

\begin{abstract}
We present an analysis and simulation of the non-paraxial nonlinear Schrödinger equation. Exact general relations describing energy flow conservation and transformation invariance are reported, and then explained on physical grounds. New instabilities of fundamental and higher-order paraxial solitons are discovered in regimes where exact analytical non-paraxial solitons are found to be robust attractors. Inverse-scattering theory and the known form of solutions are shown to enable the prediction of the characteristics of nonparaxial soliton formation. Finally, analysis of higher-order soliton break up due to non-paraxial effects reveals features that appear to be of a rather general nature.
\end{abstract}

\section{Introduction}

Spatial solitons are self-trapped beams that have innate appeal as binary elements in future optical information processing and storage devices [1-3]. They have been studied experimentally in different Kerr-like media [4-7] and analyses are usually based on the paraxial nonlinear Schrödinger equation (NSE), which is analytically solvable using inverse scattering theory [8]. In a more general context, NSE solitons have underpinned the understanding of nonlinear beams in a wide range of physical systems, including those in which higher-order effects come into play. However, in the progressive miniaturization of optical devices, the paraxial approximation will ultimately be violated when the optical wavelength is no longer negligible in comparison to the beam width.

In a previous work [9], we derived the non-paraxial nonlinear Schrödinger equation (NNSE) to give a more complete description of beam propagation in Kerr media. The first exact analytical non-paraxial soliton solution was presented and we explored its underlying mathematical and physical geometry through

$\dagger$ Permanent address: Photonics and Nonlinear Science Group, Joule Physics Laboratory, School of Sciences, University of Salford, Salford M5 4WT, UK 
dispersion relations and approximations. This work also uncovered other more general situations in which the paraxial approximation breaks down. One example is when a soliton beam propagates at a significant angle to the reference axis or, equivalently, when two or more soliton beams interact at such an angle. This latter configuration arises in the proposed use of spatial solitons as multi-port switching devices [10]. However, to date, the mathematical description of such devices has necessarily been approximate since it has been limited by assumptions of paraxiality [10]. The character of non-paraxiality in linear beam propagation is best summarized in terms of spatial spectra. A single ultra-narrow beam and the multiplexing of several broad beams are two contexts where high spatial wavenumbers arise by different means. In the first case, a broad angular spectrum results from the spatial transform of the beam profile, whereas the second configuration involves beams with individually narrow spectra but whose collective description can span a broad spatial frequency range. Precisely the same ideas are applicable to the nonlinear evolution of beams, though additional considerations also need to be made.

In this paper, we present new exact analytical properties of the non-paraxial evolution equation and then address questions relating to the propagation stability of paraxial and non-paraxial solutions. A generalized conservation law is reported that has both fundamental and practical implications. The relations describing the transformation invariance of the NNSE are then presented. These transformations can be applied to derive generalizations of exact nonlinear solutions and are shown to arise from a combination of physically meaningful operations. In our earlier work [9], we presented exact solutions but neither their basins of attraction nor their stability were examined. Our knowledge of these solutions, and of properties of the NNSE has permitted, for the first time, the testing of existing non-paraxial beam propagation methods. Here, we report results from simulations that use two new numerical algorithms that have been rigorously tested using exact analyses. We firstly report on the stability and dynamics of off-axis paraxial and nonparaxial fundamental solitons. Both our earlier analyses and inverse scattering theory prove to be useful tools for predicting the long-term states. The stability of higher-order paraxial solitons and on-axis non-paraxial solitons are then addressed.

\section{Exact analytical properties}

We consider the scalar field envelope $u(\xi, \zeta)$ of a continuous-wave (cw) beam which evolves according to the NNSE

$$
\kappa \frac{\partial^{2} u}{\partial \zeta^{2}}+i \frac{\partial u}{\partial \zeta}+\frac{1}{2} \frac{\partial^{2} u}{\partial \xi^{2}}+|u|^{2} u=0
$$

where $\zeta$ and $\xi(z$ and $x$ ) are the scaled (unscaled) longitudinal and transverse coordinates, respectively, and

$$
\zeta=\frac{z}{L_{\mathrm{D}}}, \quad \xi=\frac{2^{1 / 2} x}{w_{0}}, u(\xi, \zeta)=\left(\frac{k n_{2} L_{\mathrm{D}}}{n_{0}}\right)^{1 / 2} A(\xi, \zeta) .
$$

$w_{0}$ is a transverse scale parameter which can be considered as equivalent to the waist of a (reference) Gaussian beam with diffraction length $L_{\mathrm{D}}=k w_{0}^{2} / 2$. 
$k=n_{0} \omega / c, n_{0}$ is the linear refractive index, $n_{2}$ is the Kerr coefficient, $A(\xi, \zeta)$ is the unscaled field and $\kappa=1 /\left(k w_{0}\right)^{2}=\left(\lambda / w_{0}\right)^{2} / 4 \pi^{2} n_{0}^{2}$, where $\lambda$ is the optical wavelength. In free space, $\kappa=10^{-3}, 10^{-4}$ and $10^{-5} \mathrm{imply}$ around $10 \lambda, 32 \lambda$ and $100 \lambda$, respectively, in the full-width of the reference beam.

\subsection{Non-paraxial solitons}

We have shown [9] that the NNSE has a bright non-paraxial soliton solution given by

$$
u(\xi, \zeta)=\eta \operatorname{sech}\left[\frac{\eta(\xi+V \zeta)}{\left(1+2 \kappa V^{2}\right)^{1 / 2}}\right] \exp \left[i\left(\frac{1+2 \kappa \eta^{2}}{1+2 \kappa V^{2}}\right)^{1 / 2}\left(-V \xi+\frac{\zeta}{2 \kappa}\right)\right] \exp \left[\frac{-\mathrm{i} \zeta}{2 \kappa}\right],
$$

in which the usual amplitude and transverse velocity parameters, $\eta$ and $V$ respectively, are supplemented with the non-paraxial parameter $\kappa$. It can be seen that the width of the non-paraxial soliton is given by $\xi_{0}=\left(1+2 \kappa V^{2}\right)^{1 / 2} / \eta$. Its area is thus proportional to $\xi_{0} \eta=\left(1+2 \kappa V^{2}\right)^{1 / 2}$ and depends on both the transverse velocity and the size of the beam (through $\kappa$ ). In common with the paraxial solution, this area is defined by the initial conditions and remains conserved during subsequent propagation. Non-paraxiality also introduces a correction in the longitudinal phase of the beam; for a beam with zero transverse velocity, the soliton phase period is $\zeta_{0}=4 \pi / \eta^{2} \beta$, where $\beta$ is the solution of $\kappa \eta^{2} \beta^{2}+2 \beta=2$.

The NSE is an approximation to the NNSE as the term involving $\kappa$ is neglected. Both wave equations describe beam evolution with respect to a forward-propagating reference frame. However, while the paraxial equation neglects backward-travelling waves, it has been shown that the non-paraxial description does not entail this approximation [9]. The NSE is recovered from equation (1) under certain limiting conditions. A beam that is broad compared to the optical wavelength, $\kappa \rightarrow 0$, is an obvious choice, but this is neither a necessary nor a sufficient condition. Strictly, one has to require that $\kappa\left|\partial^{2} u / \partial \zeta^{2}\right|$ remains negligible with respect to the other terms of the NNSE. For nonlinear solutions, one needs to stipulate that the peak intensity is such that self-phase modulation leads to sufficiently slow variations along the $\zeta$ coordinate. This is in addition to the constraints that need to be placed on the spatial spectrum. In fact, all of the above considerations involve the parameter $\kappa$. This is clearly demonstrated in the case of the particular solution (3); to recover the paraxial soliton, one is required to enforce the multiple limit $\kappa \rightarrow 0, \kappa \eta^{2} \rightarrow 0$ and $\kappa V^{2} \rightarrow 0$.

\subsection{Energy flow conservation}

The NSE is exactly integrable and thus possesses an infinite number of conserved quantities. The first of these is the energy invariant; any solution $u(\xi, \zeta)$, evolving according to the NSE, satisfies

$$
\int_{-\infty}^{+\infty}|u(\xi, \zeta)|^{2} \mathrm{~d} \xi=C,
$$

where $C$ is a constant. To derive a counterpart of expression (4), that is applicable to solutions of the NNSE, one multiplies the non-paraxial wave equation by $u^{*}(\xi, \zeta)$ and integrates the real part of the resulting equation with respect to the 
transverse coordinate. Then, by writing the solution in terms of its modulus $|u(\xi, \zeta)|$ and its phase $\phi(\xi, \zeta)$, and assuming that the solution vanishes as $\xi \rightarrow \pm \infty$, one finds that

$$
\int_{-\infty}^{+\infty}\left(\frac{1}{2 \kappa}+\frac{\partial \phi(\xi, \zeta)}{\partial \zeta}\right)|u(\xi, \zeta)|^{2} \mathrm{~d} \xi=C^{\prime}
$$

where $C^{\prime}$ is a constant. In the non-paraxial case, it is thus the energy flow that is conserved. Equation (5) generalizes a previously published result for which only fast on-axis phase variations were taken into account [11, 12]. The appearance of $1 / 2 \kappa$ in the energy flow expression is due to the phase reference assumed in the derivation of the NNSE. A fast phase term exp $(\mathrm{i} k z)$ is implicit in the solutions and this becomes $\exp (i \zeta / 2 \kappa)$ in the normalized reference frame. The expression inside the bracket in equation (5) is thus the $\zeta$ derivative of the total phase in scaled coordinates.

Since both forward- and backward-travelling waves can be described by the non-paraxial equation, we note that a solution with $\phi(\xi, \zeta)=-\zeta / 2 \kappa+\phi_{0}$, where $\phi_{0}$ is a constant, is a nonlinear standing wave. As one would expect, the energy flow in this case is zero. For solutions that have phase of the form $\alpha \zeta+\phi_{0}$, where constant $\alpha \neq-1 / 2 \kappa$, the longitudinal phase derivative is constant and the paraxial energy invariant is conserved. This is the case for stationary solutions such as the $V=0$ fundamental non-paraxial soliton. When $V \neq 0$, the energy flow for a non-paraxial soliton is found to be $\eta\left(1+2 \kappa \eta^{2}\right)^{1 / 2} / \kappa$ and is thus independent of the transverse velocity. This is to be expected since, for fixed $\eta$ and $\kappa$, expression (3) can be considered to describe a single forward-propagating beam whose energy flow should be independent of the particular orientation of the coordinate axes.

\subsection{Transformation invariance}

The paraxial NSE is known to be invariant under the Galilean transformation [13]; when the coordinates are changed according to

$$
\xi=\xi^{\prime}+V \zeta^{\prime}, \quad \zeta=\zeta^{\prime},
$$

one is also required to transform the field as

$$
u(\xi, \zeta)=\exp \left[\mathrm{i}\left(V \xi^{\prime}+\frac{1}{2} V^{2} \zeta^{\prime}\right)\right] u^{\prime}\left(\xi^{\prime}, \zeta^{\prime}\right)
$$

to preserve the form of the equation. Instead of applying these relations to the evolution equation, one can apply them to known solutions. This provides a means of generating general solutions from particular ones that can sometimes be more straightforward to find. We have derived the transformations under which the NNSE is invariant:

$$
\xi=\frac{\xi^{\prime}+V \zeta^{\prime}}{\left(1+2 \kappa V^{2}\right)^{1 / 2}}, \quad \zeta=\frac{-2 \kappa V \xi^{\prime}+\zeta^{\prime}}{\left(1+2 \kappa V^{2}\right)^{1 / 2}}
$$

and

$$
u(\xi, \zeta)=\exp \left[\mathrm{i}\left(\frac{V \xi^{\prime}}{\left(1+2 \kappa V^{2}\right)^{1 / 2}}+\frac{1}{2 \kappa}\left(1-\frac{1}{\left(1+2 \kappa V^{2}\right)^{1 / 2}}\right) \zeta^{\prime}\right)\right] u^{\prime}\left(\xi^{\prime}, \zeta^{\prime}\right) .
$$

The Galilean transformation is, of course, recovered in the appropriate paraxial limit. Expressions (8) and (9) can be used to introduce, or to remove, the transverse 
velocity dependence of a non-paraxial solution, and thus to effect a rotation of this solution in the $(\xi, \zeta)$ plane. Considering the non-paraxial soliton solution (3), a beam with zero transverse velocity defines a stripe of light of width $1 / \eta$. When $V \neq 0$, this same stripe will lie at an angle $\theta$ to the longitudinal axis. One thus expects the beam width (at fixed $\zeta$ ) to be increased. The correspondence between this physical rotation and the form of the non-paraxial soliton yields $\sec \theta=\left(1+2 \kappa V^{2}\right)^{1 / 2}$ and fixes the relation between $\theta$ and $V$.

It should be stressed that the absence of a beam-broadening factor in the paraxial soliton is unphysical. On the other hand, the non-paraxial transformation (8) can be decomposed into three physical steps. Firstly, a solution is written in terms of the original (unscaled) coordinates. Then, a rotation of angle $\theta$ is introduced. Finally, the solution is transformed back to scaled units. This yields an alternative form for (8), in terms of the angle of rotation,

$$
\left[\begin{array}{l}
\xi \\
\zeta
\end{array}\right]=\left[\begin{array}{cc}
\cos \theta & \frac{1}{(2 \kappa)^{1 / 2}} \sin \theta \\
-(2 \kappa)^{1 / 2} \sin \theta & \cos \theta
\end{array}\right]\left[\begin{array}{l}
\xi^{\prime} \\
\zeta^{\prime}
\end{array}\right] .
$$

There is also an additional phase term in (9) that accounts for the factor exp (ikz) introduced by transforming to the forward-propagating reference frame.

\section{Initial value problems}

Numerical studies are needed to address important questions regarding the stability of nonlinear solutions and whether they can be generated from arbitrary initial conditions. Our exact non-paraxial solution and the energy flow conservation law provide, for the first time, a framework for testing existing non-paraxial beam propagation methods. Indeed, distinct methods tend to yield quantitatively different results [14] and, to obtain accurate results, we found it necessary to derive new algorithms.

Firstly, we developed a generalization of the Feit-Fleck method [15]. After analysing their approach, we proposed a modified evolution operator. Both this and the exact propagation operator were expanded in power series of the diffraction, $\partial^{2} / \partial \xi^{2}$, and the nonlinear, $|u|^{2}$, operators; the leading order error terms were then corrected. This allowed for an accurate computation of off-axis non-paraxial soliton propagation, provided that the peak beam intensity was $O(1)$. Secondly, a complementary method, based on a finite-difference scheme, was derived to study the propagation of higher-order paraxial solitons. In this case, the restriction on the peak intensity of the solution was relaxed. A detailed account of these algorithms, including comparisons with well-known methods and error analyses, will be presented elsewhere. In this paper, we focus on the mathematical and physical consequences of non-paraxiality.

\subsection{Off-axis non-paraxiality}

In this subsection, we study non-paraxial soliton formation from the initial condition $u(\xi, 0)=\operatorname{sech}(\xi) \exp \left(-i S_{0} \xi\right)$, which corresponds to an exact paraxial soliton with $\eta=1$ and $V=S_{0}$. One may suspect that the paraxial approximation could fail for large $S_{0}$, since the spatial spectrum of the propagating beam is then highly asymmetrical. For beam widths of at least a few optical wavelengths 
$(\kappa \ll 1)$, and for $\kappa \eta^{2} \ll 1$, the slope of the transverse phase profile of the nonparaxial soliton is given by

$$
S=V\left(\frac{1+2 \kappa \eta^{2}}{1+2 \kappa V^{2}}\right)^{1 / 2} \simeq \frac{V}{\left(1+2 \kappa V^{2}\right)^{1 / 2}}=\frac{\sin \theta}{(2 \kappa)^{1 / 2}} .
$$

Both the propagation angle $\theta$ and the slope of the phase profile are constant during propagation. $\kappa \eta^{2} \ll 1$ implies, in this case, that non-paraxiality arises primarily from the angle that the beam makes to the $\zeta$ axis. Then, by applying transformations (8) and (9) and examining the beam in the $\theta$ direction, the initial condition can be considered as a perturbed paraxial soliton (its width having been reduced by a factor of $\left.\left(1+2 \kappa V^{2}\right)^{1 / 2}\right)$. Thus, the long term evolution of the soliton beam can be predicted by using (paraxial) inverse scattering techniques [13].

Figure 1(a) shows the evolution of the peak amplitude of the beam $|u|_{m}$ for $\kappa=10^{-3}$ and for three values of $S_{0}$ (giving $\theta=12.9^{\circ}, 26.6^{\circ}$ and $42.1^{\circ}$ ). Horizontal lines show the predicted asymptotic values of $|u|_{m}$. It can be seen that the beam parameters undergo decaying oscillations and approach the predicted limit value in a formally analogous fashion to perturbed paraxial solitons with $V=0$. For $\kappa=10^{-4}$ and after multiplying the previous values of $S_{0}$ by $10^{1 / 2}$ (broader beams launched at approximately the same angles), we found that the results obtained were almost identical, as expected for $\kappa \eta^{2} \ll 1$. However, the physical length scalings for the two values of $\kappa$ are different and a more rapid evolution to a stationary non-paraxial soliton profile occurs for narrower beams. Figure $1(b)$ shows the evolution of the beam area $A$ (as computed from the numerical data). The non-paraxial soliton that ultimately results is determined by the initial condition. Its area depends on $S_{0}$ and is given by the beam broadening factor, $1 / \cos \theta$, of the appropriate solution (plotted as a horizontal line).

\subsection{On-axis non-paraxiality}

We now consider the initial condition $u(\xi, 0)=N$ sech $(\xi)$, where $N=2,3,4, \ldots$, that, in the paraxial limit, leads to higher-order solitons propagating along the $\zeta$ axis. These solutions are multi-soliton quasi-bound states (soliton superpositions with zero binding energy) in which the beam profile undergoes periodic focusing and defocusing stages. The NSE solitons can be of arbitrarily high order and this can lead to extremely narrow beams (and hence very wide symmetric spatial spectra) at certain propagation distances. During such narrowing, non-paraxial effects can play an important role and one could expect that this places a physical limit on the order of the soliton that can be supported.

While $N \gg 1$ and $\kappa \simeq 10^{-5}$ is a particular physical context of interest (i.e. quasi-paraxial input leading to non-paraxial effects), for a clearer picture of the beam evolution, we highlight the case of $N=3$ and a larger value of $\kappa$. In figure 2, we plot the evolution of the peak amplitude for three levels of non-paraxiality. As $\kappa$ increases from zero, it is found that non-paraxiality leads to changes in the periodicity of the beam and that higher-order soliton-like beams can propagate for relatively long distances. However, for larger values of $\kappa$, this pattern breaks down completely. Figure 3 gives a more detailed picture of this latter case, where snapshots of the transverse intensity profile are plotted. It can be seen that the multi-soliton beam is destroyed during the initial stages of propagation. This results in distinct non-paraxial soliton beams that propagate stably and travel 


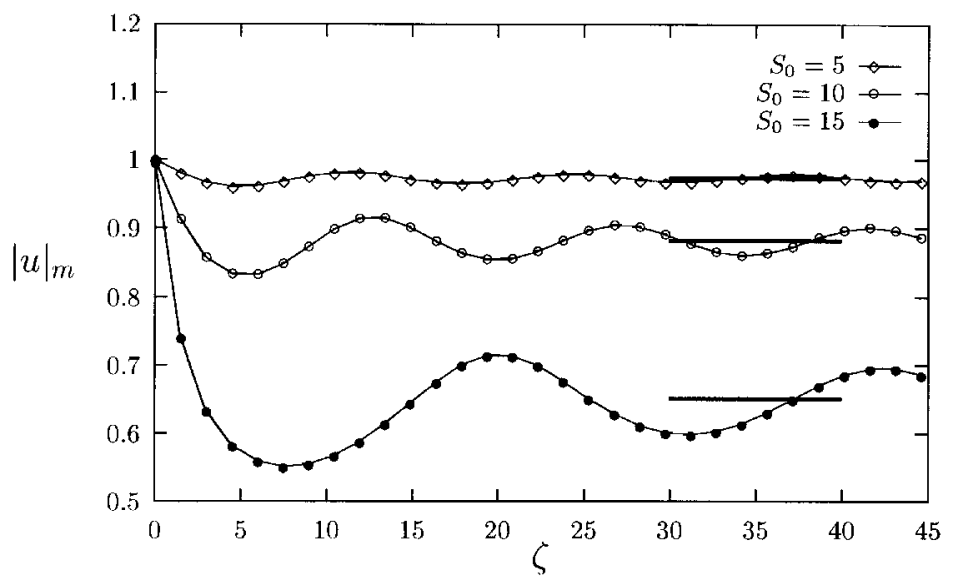

(a)

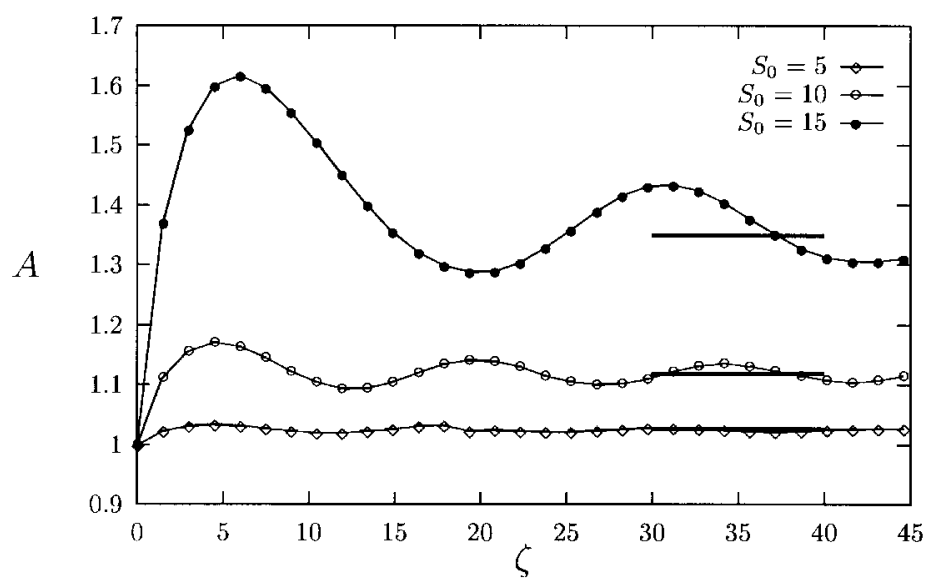

(b)

Figure 1. Formation of non-paraxial solitons from paraxial soliton initial conditions. Evolution of $(a)$ peak amplitude and $(b)$ beam area for a launched beam with profile $u(\xi, 0)=\operatorname{sech}(\xi) \exp \left(-\mathrm{i} S_{0} \xi\right)$. Horizontal lines display the long term evolution values predicted from analyses. $\left(\kappa=10^{-3}\right.$ and the three values of $S_{0}$ considered are shown in the inset.)

either along the $\zeta$ axis or escape from this axis as pairs of equal amplitude solitons with opposite transverse velocities.

Similar behaviour has in fact been reported for higher-order solitons perturbed by two-photon absorption [16] and the mathematical description of that problem is found to lend insight into the effects described here. In figure 4, we map out the eigenvalues of the direct-scattering problem as the beam evolves [17]. Focusing stages are found to introduce shifts in the amplitudes of the constituent solitons that, in turn, result in a distortion of the periodicity of the beam [16]. Moreover, higher-order soliton splitting is found to exhibit a similar bifurcation structure to that reported in [16] and break-up occurs when two of the constituent solitons become degenerate. Each of the above characteristics is thus likely to be of a more 


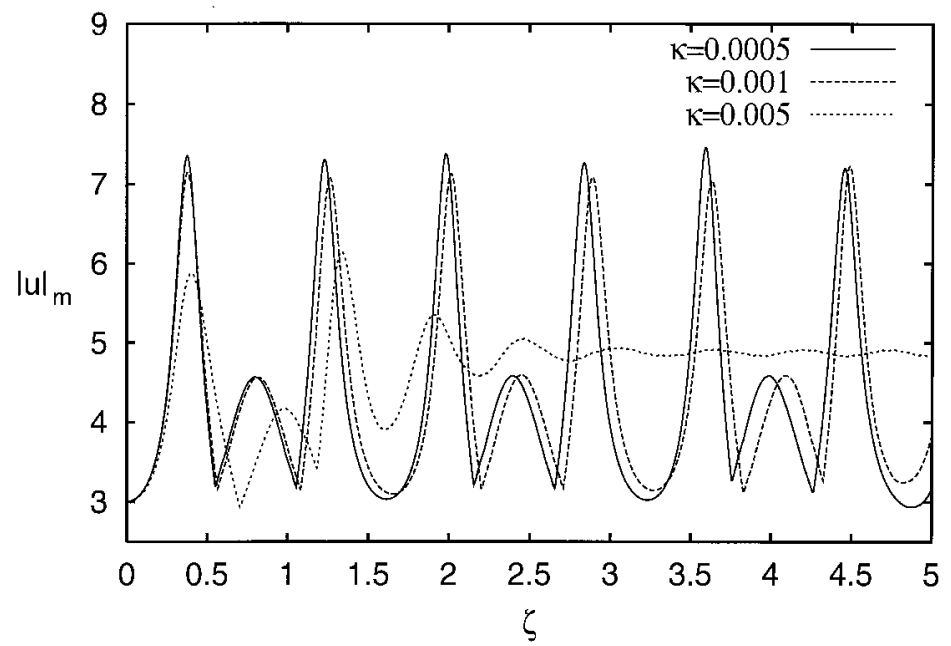

Figure 2. Peak amplitude of $N=3$ soliton beams showing the modification of the soliton period and then the breakdown of periodicity as $\kappa$ varies from 0.0005 to 0.005 .

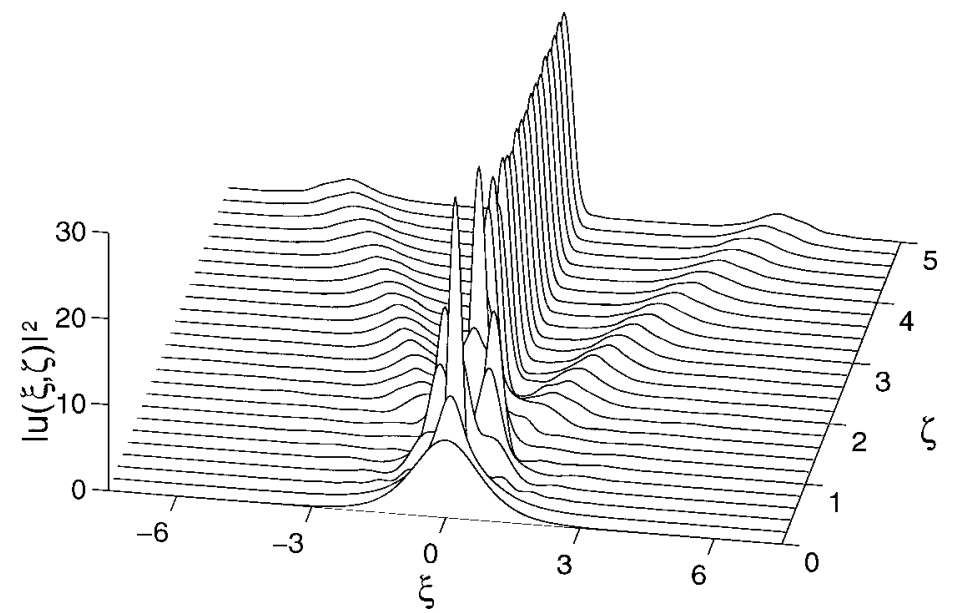

Figure 3. Transverse intensity profiles showing the splitting of a $N=3$ soliton due to non-paraxial effects $\left(\kappa=5 \times 10^{-3}\right)$.

general nature. In particular, an exclusion principle for soliton beams appears to apply, in which solitons of the same energy cannot co-exist at the same location in transverse space. Such a principle could prove to be extremely useful in the study, or the manipulation, of large ensembles of solitons.

Finally, since higher-order soliton propagation presents a reasonably severe test of the accuracy of a numerical algorithm, we use this example to illustrate the distinctness of the paraxial and non-paraxial conserved quantities and the accuracy of our numerical technique. Figure 5 shows the evolution of the integrated beam intensity and the energy flow during the $N=3$ soliton splitting process. Note that the vertical scale has been greatly magnified in this plot. The data highlights both 

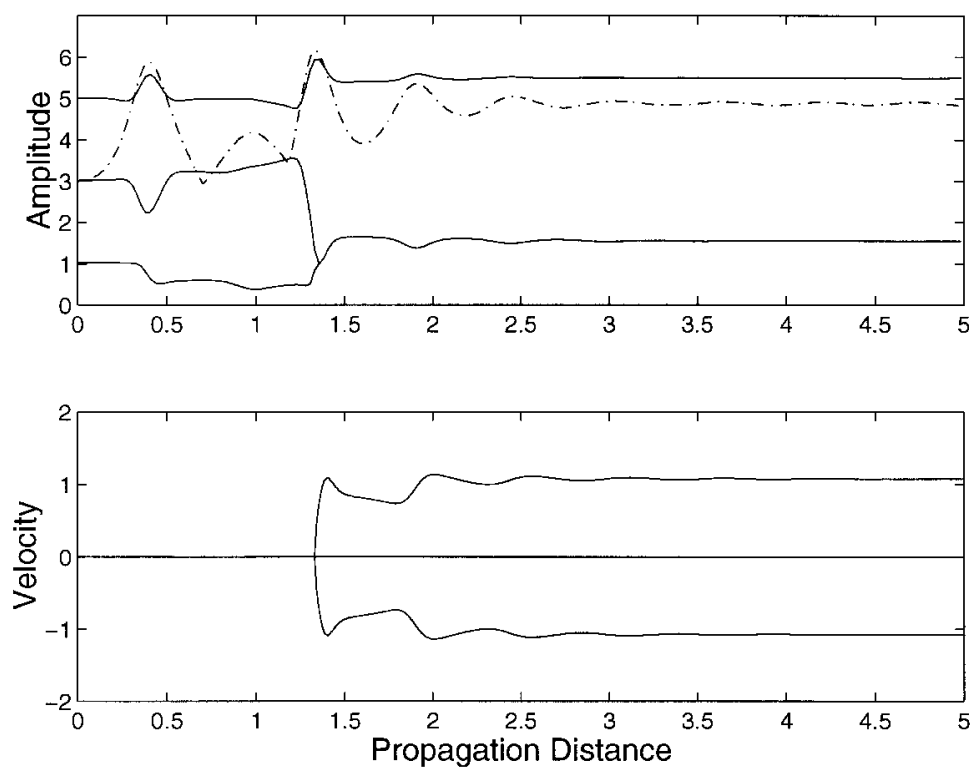

Figure 4. Amplitudes and velocities of the constituent solitons for the data shown in figure 3. The dashed curve gives the peak amplitude of the total field.

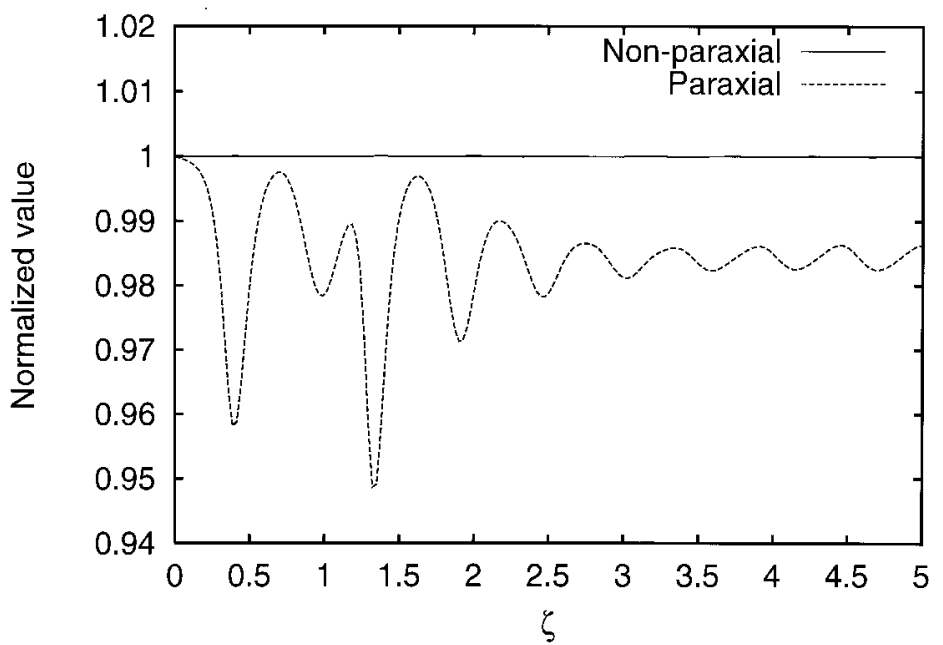

Figure 5. Evolution of the paraxial energy invariant and the non-paraxial energy flow during the break-up of a $N=3$ paraxial soliton.

the integrity of the computations reported and the breakdown of the paraxial conservation law.

\section{Conclusions}

We have presented and explained new exact analytical properties of the non-paraxial nonlinear Schrödinger equation: the energy flow conservation law and the relations governing transformation invariance. Simulations demonstrate 
the inappropriateness of both fundamental and higher-order paraxial soliton solutions in the non-paraxial regime and, instead, that non-paraxial solitons act as robust attractors. Features uncovered in the analysis of perturbation-induced break-up of higher-order solitons appear to be generic in character. We have also shown that non-paraxial analysis and/or inverse-scattering theory can be used to predict the parameters of soliton formation beyond the paraxial regime. Given the fundamental role that paraxial solitons have played in nonlinear optics, the explicit mathematical and computational features reported in this work are also expected to have important implications for analysis and simulation of non-paraxial solitons in systems of higher dimension and when additional higher-order effects are taken into account.

\section{Acknowledgments}

This research was supported in part by EPSRC grant number GR/L90583 and by contract CICYT PB97-0487.

\section{References}

[1] McDonald, G. S., and Firth, W. J., 1990, J. mod. Optics, 37, 613.

[2] McDonald, G. S., and Firth, W. J., 1990, J. opt. Soc. Am. B, 7, 1328.

[3] McDonald, G. S., and Firth, W. J., 1993, J. opt. Soc. Am. B, 10, 1081.

[4] Maneuf, S., and Reynaud, F., 1988, Optics Commin., 66, 325.

[5] Maneuf, S., Desallly, R., and Froehly, C., 1988, Optics Commun., 65, 193.

[6] Aitchison, F., Weiner, A., Silberberg, Y., Oliver, M., Jackel, J., Leaird, D., Vogel, E., and Sмith, P., 1991, J. opt. Soc. Am. B, 8, 1290.

[7] Shalaby, M., Reynaud, F., and Barthelemy, A., 1988, Optics Commun., 65, 193.

[8] Zaharov, V. E., and Shabat, A. B., 1972, Sov. Phy. JETP, 34, 62.

[9] Chamorro-Posada, P., McDonald, G. S., and New, G. H. C., 1998, J. mod. Optics, 45, 1111.

[10] Miller, P. D., and Akhmediev, N. N., 1996, Phys. Rev. E, 53, 4098.

[11] Akhmediev, N., Ankiewicz, A., and Soto-Crespo, J. M., 1993, Optics Lett., 18, 411.

[12] Soto-Crespo, J. M., and Akhmediev, N., 1993, Optics Commun., 101, 22.

[13] Satsuma, J., and Yajima, N., 1974, Progr. Theor. Phys., Osaha, Supplement, 55, 284.

[14] Chi, S., and Guo, Q., 1995, Optics Lett., 20, 1598.

[15] Feit, M. D., and Fleck, J. A., 1988, J. opt. Soc. Am. B, 7, 633.

[16] Afanasjev, V. V., Aitchison, J. S., and Kivshar, Y. S., 1995, Optics Commun., 116, 331.

[17] Chamorro-Posada, P., McDonald, G. S., New, G. H. C., and Fraile-Pelaez, F. J., 1999, IEEE Trans. Magn., 35, 1558. 